\title{
Low-energy dipole response of exotic nuclei ${ }^{\star}$
}

\author{
Thomas Aumann ${ }^{1,2, \text { a }}$ \\ 1 Institut für Kernphysik, Technische Universität Darmstadt, Schlossgartenstraße 9, D-64289 Darmstadt, Germany \\ 2 GSI Helmholtzzentrum für Schwerionenforschung, Planckstr. 1, D-64291 Darmstadt, Germany
}

Received: 3 May 2019 / Revised: 13 August 2019

Published online: 3 December 2019

(c) The Author(s) 2019. This article is published with open access at Springerlink.com

Communicated by N. Alamanos

\begin{abstract}
The electric dipole response of neutron-rich nuclei is discussed from an experimental perspective using selected examples. After introducing the main experimental method, which is relativistic Coulomb excitation in conjunction with invariant-mass spectroscopy, the response of neutron-rich nuclei is discussed separately for light halo nuclei and heavier neutron-rich nuclei. Finally, the perspective for constraining the equation of state of neutron-rich matter close to saturation density from measurements of the dipole response of neutron-rich nuclei is discussed.
\end{abstract}

\section{Introduction}

The multipole response of nuclei is dominated by collective excitation modes, the giant resonances [1]. The electric monopole, dipole, and quadrupole giant resonances have been studied extensively for stable nuclei. For not too light nuclei, they exhibit a characteristic Lorentzianlike shape with smoothly varying parameters as a function of the mass number $A$, and exhaust around $100 \%$ of the classical sum rules [2]. The giant dipole resonance (GDR) is understood as a harmonic vibration of neutrons versus protons. Indeed, the second phonon of this harmonic vibration has been found experimentally at an excitation energy close to twice the excitation energy of the GDR [3].

The question of how the dipole response of a nucleus changes when going away from the valley of stability has attracted a lot of interest since the availability of radioactive beams and the discovery of halo nuclei $[4,5]$. But not only for very loosely bound neutron-rich nuclei at the drip line substantial changes in the dipole response are expected. The very different filling of nuclear levels leads to very different Fermi energies for neutrons and protons which should manifest itself in the excitation spectra. In general, theoretical investigations suggested a redistribution of the dipole strength towards lower energy below the GDR. Microscopic calculations predicted the development of a new collective excitation mode for neutron-rich nuclei at around 8 to $10 \mathrm{MeV}$, the pygmy dipole resonance

\footnotetext{
* Contribution to the Topical Issue "Giant, Pygmy, Pairing Resonances and Related Topics" edited by Nicolas Alamanos, Ricardo A. Broglia, Enrico Vigezzi.

${ }^{a}$ e-mail: t.aumann@gsi.de
}

(PDR). An overview on the theoretical efforts to understand this phenomenon can be found in the review article of Paar et al. [6]. It is expected, that the strength of this excitation mode increases along isotopic chains with neutron excess [6], and in particular when the binding of the excess neutrons becomes significantly smaller, e.g., beyond a closed shell [7].

The existence of a PDR has also been discussed for stable nuclei with neutron excess but symmetric Fermi surface and has triggered substantial experimental effort. So far, however, no clear picture on a systematic behaviour of the low-lying dipole strength emerged.

In this article, an overview on different aspects of the electric dipole response of neutron-rich nuclei is given using selected examples without aiming at providing an exhaustive review of the experimental work performed so far. More complete summaries on the experimental situation including stable nuclei can be found in the review articles by Savran et al. [8] and Bracco et al. [9], and with a focus on neutron-rich unstable nuclei in ref. [10].

Concerning the dipole response of exotic nuclei, the findings from the existing experimental data can be summarized as follows. It is meanwhile well established that, in addition to the giant dipole resonance, low-lying dipole strength develops in neutron-rich nuclei, which is related to the neutron excess. Tentatively, we may distinguish three characteristic kinds of the low-lying dipole strength:

1) Threshold dipole strength of weakly bound oneneutron halo nuclei, which is understood as a nonresonant transition related to the spatial extension of the single-particle wave function of the halo neutron. The shape and magnitude of the strength carries information on the valence-nucleon shell structure. 
2) Low-lying dipole strength of two-neutron halo nuclei, which might be of resonant or non-resonant character. The strength is sensitive to the correlations among the valence neutrons and the spatial extension of the halo. This implies also sensitivity to the angular momentum components of the valence neutron wave function.

3) In case of heavier neutron-rich nuclei, the low-lying dipole strength is often referred to as "pygmy" resonance, which is commonly understood in terms of the excitation of less-well bound valence neutrons, and which might be interpreted as a vibration of the neutron skin against the core.

Collective nuclear excitation modes carry also information on the properties of nuclear matter. Since collective dipole modes are related to the separation of neutrons and protons, they carry information on the symmetry energy, i.e., the equation of state for asymmetric nuclear matter. Theoretical studies showed, that in particular the dipole polarizability of a neutron-rich nucleus can be directly related to the symmetry energy and its slope, i.e. the neutron pressure, close to saturation density $[11,12]$. Another nuclear ground-state observable which correlates well with the symmetry-energy parameters is the neutronskin thickness of neutron-rich nuclei. Obtaining experimental constraints on these quantities is of particular interest. Since a large fraction of the volume of a neutron star has densities around nuclear saturation density, constraints obtained from nuclear observables such as the dipole polarizability translate directly into constraints on the mass-radius relation of neutron stars.

\section{Experimental method}

The ideal probe to study the nuclear dipole response is real-photon absorption and scattering, which is, however, difficult to apply to short-lived nuclei. Heavy-ion induced electromagnetic excitation at relativistic beam energies is an alternative, which has been established as a spectroscopic tool over the past 30 years. The rapidly varying Lorentz-contracted electric field seen by the projectile passing a high- $Z$ target translates into an equivalent photon spectrum with correspondingly high frequencies. The excitation-energy window accessible depends on the beam energy and can be estimated by the adiabatic cutoff energy $E_{\gamma, \max }=\gamma \beta \hbar c / b$, where the Lorentz factor $\gamma$ and the impact parameter $b$ enter. For a collision of, e.g. ${ }^{132} \mathrm{Sn}+{ }^{208} \mathrm{~Pb}$ at $800 \mathrm{MeV} / \mathrm{u}$ at $b=15 \mathrm{fm}$, the highest reachable excitation energy lies around $20 \mathrm{MeV}$ according to this estimate, which just covers the GDR region located at around $15 \mathrm{MeV}$. The cross section $\sigma_{\pi \lambda}$ for electromagnetic excitation is directly related to the photo-absorption cross section and thus to the matrix element $B(\pi \lambda)$ for transitions of multipolarity $\pi \lambda$, and can be written as a product of the photo-nuclear cross section $\sigma_{\gamma}^{\pi \lambda}$ and the number $N^{\pi \lambda}$ of equivalent photons [13]:

$$
\sigma^{\pi \lambda}=\int_{b_{\min }}^{\infty} P(b) 2 \pi b \mathrm{~d} b=\int N_{\pi \lambda}(E) \sigma_{\gamma}^{\pi \lambda}(E) \frac{\mathrm{d} E}{E},
$$

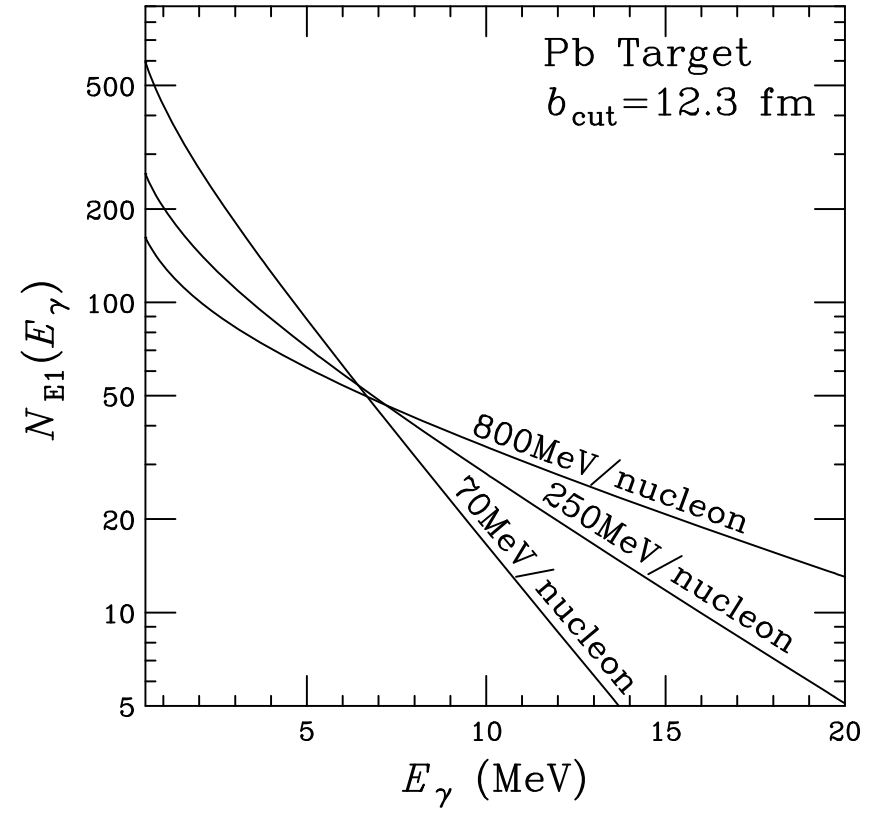

Fig. 1. $E 1$ virtual-photon spectrum for projectiles at 70,250 and $800 \mathrm{MeV} / \mathrm{u}$ passing a $\mathrm{Pb}$ target with impact parameters $b>b_{\min }=12.3 \mathrm{fm}$. The figure is taken from ref. [10].

where $E$ denotes the excitation energy and $N_{\pi \lambda}(E)$ the number of equivalent photons with multipolarity $\pi \lambda$ and energy $E . N_{\pi \lambda}(E)$ results from an integration over $b$ from a minimum impact parameter $b_{\text {min }}$ which corresponds to the sum of projectile and target radii below which nuclear absorption dominates. A more elaborated method is to calculate the nuclear absorption using the eikonal approximation with realistic density distributions for projectile and target as an input [14].

The equivalent or virtual photon spectrum $N_{\pi \lambda}(E)$ depends strongly on the beam energy. Figure 1 shows as an example the $E 1$ spectrum for a $\mathrm{Pb}$ target and $b_{\min }=12.3 \mathrm{fm}$ for three different beam energies. It is clearly visible that high beam energies are required in order to extract the full dipole strength of a nucleus up to $30 \mathrm{MeV}$. It is interesting to note that the three curves cross each other at around 6 to $7 \mathrm{MeV}$. For low-energy excitations, lower beam energies might thus be advantageous.

Depending on the case, $E 1, E 2$, and $M 1$ transitions might contribute non-negligibly to the cross section. Equations for the computation of $N^{\pi \lambda}$ can be found in ref. [13]. The lower the beam energy, E2 (M1) photons become more (less) abundant compared to $E 1$. In the ultrarelativistic limit $(\beta=1, \gamma \rightarrow \infty)$, the virtual-photon spectra of all multipolarities become identical $\left(N^{\pi \lambda}=N^{E 1}\right)$, i.e., the generated electromagnetic pulse corresponds to an incident plane wave as it is the case for a real-photon beam. In that case, eq. (1) can be used without distinguishing multipolarities and the Coulomb cross section becomes directly proportional to the photo-nuclear cross section. The formalism also coincides in that limit with the equivalent-photon method formulated by Fermi, Weizsäcker, and Williams [15-17]. 
Equation (1) holds for small excitation probabilities $P(b)$ when only one virtual photon is exchanged. For collisions of heavy projectiles and heavy targets at energies around $1 \mathrm{GeV} / \mathrm{u}$, however, the cross section for GDR excitation is in the order of a barn, and the excitation probability at close impact becomes as large as 0.3 . The effect of two-photon exchange can be incorporated in the formalism, which leads to a slight reduction of the cross section in the GDR region. The second-order process leads to the excitation of the two-phonon GDR, which has been discovered in the beginning of the 1990s and which was studied by making use of exactly this property of relativistic Coulomb excitation [3].

Finally, it is worth noting that the equivalent-photon method as formulated by Bertulani and Baur [13], introducing different multi-polarities explicitly, is based on the same theoretical approximations as the theory for relativistic Coulomb excitation developed by Winther and Alder [18] and yields the same results for the cross sections.

In order to extract the dipole-strength distribution from the measured cross section, the contributions by the different multipolarities as well as nuclear excitation have to be separated. In principle, this is possible by making use of the cross-section dependencies on beam energy, target, and scattering angle. Since the magnetic dipole is suppressed compared to real-photon absorption, $M 1$ contributions can be safely neglected in most cases. Electric quadrupole excitations, however, are enhanced at typical beam energies used at the fragmentation facilities. The contribution in the energy region of the giant quadrupole resonances (GQR) is in the order of $10 \%$. The very different energy dependence of the cross sections for $E 1$ and $E 2$ excitation can be used to separate the two contributions. However, experiments did not make use of this so far. Often $E 2$ excitations are taken into account in the analysis by calculating the cross section according to the systematics for stable nuclei. Since the contribution is only $10 \%$, such a correction should be sufficient given the precision of the existing data.

The nuclear contribution to the cross section is most often estimated by a measurement with a light target, where electromagnetic excitation is negligible. The so obtained cross section is usually scaled by an empirical factor or by a theoretical ratio for nuclear excitation with both targets before subtracting from the measurement with the $\mathrm{Pb}$ target. Possible nuclear-Coulomb interference effects are neglected. An alternative method is to measure precisely the scattering-angle distribution. At small scattering angles, the Coulomb cross section is enhanced compared to the nuclear contribution, which might then be neglected at small angles. Both methods are obviously not exact. The next-generation experiments aiming at higher precision have to carefully estimate possible uncertainties. The measurement of a series of targets can give important additional information to cross-check the analysis. The electromagnetic cross section scales approximately with $Z^{2}$, while the nuclear cross section scales only with $A^{1 / 3}$ (for peripheral reactions as relevant here).
Since the equivalent-photon spectrum is continuous, the excitation energy has to be determined by a kinematical complete measurement of the decay products. A typical setup for such a measurement is shown in fig. 2 . Since most often a radioactive beam is used, the incoming ions have to be identified on an event-by-event basis and tracked onto the target position. The upper left inset shows an example where a cocktail beam containing light neutron-rich nuclei was impinging on the target. In the example of the GSI setup as shown schematically in fig. 2, the target is surrounded by a $4 \pi \gamma$ calorimeter (Crystal Ball) consisting of 160 individual NaI detectors. In the case of heavier nuclei or neutron-rich nuclei as discussed here, the excited projectile decays via neutron and/or photon emission. Photons are detected in Crystal Ball. A high efficiency is mandatory for the experiments discussed here, since several photons might be emitted in the decay. The sum energy of all photons will be needed for reconstructing the excitation energy. Neutrons are detected close to zero degree in an angular range of $\pm 80 \mathrm{mrad}$, which is defined by the detector size of $2 \times 2 \mathrm{~m}^{2}$ placed at around $12 \mathrm{~m}$ distance from the target, and by the gap of the dipole magnet (ALADIN), which serves to bend the charged fragments. This rather limited angular range is sufficient due to the kinematical forward focussing at the relativistic beam energies used. Neutrons evaporated after GDR excitation, for instance, have Maxwellian-like distributed kinetic energies around few $\mathrm{MeV}$. At $500 \mathrm{MeV} / \mathrm{u}$, for instance, the angular acceptance of $\pm 80 \mathrm{mrad}$ corresponds to a $100 \%$ acceptance for neutrons with kinetic energies up to $4 \mathrm{MeV}$ in the projectile frame. The acceptance smoothly decreases for higher neutron energies, which is taken into account in the data analysis. The neutron momenta are reconstructed from position and time-of-flight measurements with $1 \sigma$ resolutions of $3 \mathrm{~cm}$ and $250 \mathrm{ps}$, respectively. Charged fragments are deflected by the dipole field and their trajectories are reconstructed from position measurements (Fiber detectors in fig. 2) and the magnetic rigidity is determined. Together with energy-loss and time-of-flight measurements (ToF wall) fragments are identified on an event-by-event basis. The right lower inset of fig. 2 shows an example for the identification of fragments emerging from reactions of a ${ }^{20} \mathrm{O}$ beam.

The kinematical complete measurement of the final state as sketched above provides after identification and momentum measurement of all decay products the information necessary to reconstruct the excitation energy of the projectile prior to decay by analyzing the invariant mass $M$ through

$$
M^{2}=\left(\sum_{i} P_{i}\right)^{2}=\left(m_{P}+E\right)^{2}
$$

where $P_{i}$ denote the four-momenta of the dissociation products, $m_{P}$ the projectile rest mass, and $E$ the excitation energy of the projectile. The residual heavy fragment is not necessarily in its ground state after neutron emission, and its remaining excitation energy will be released 

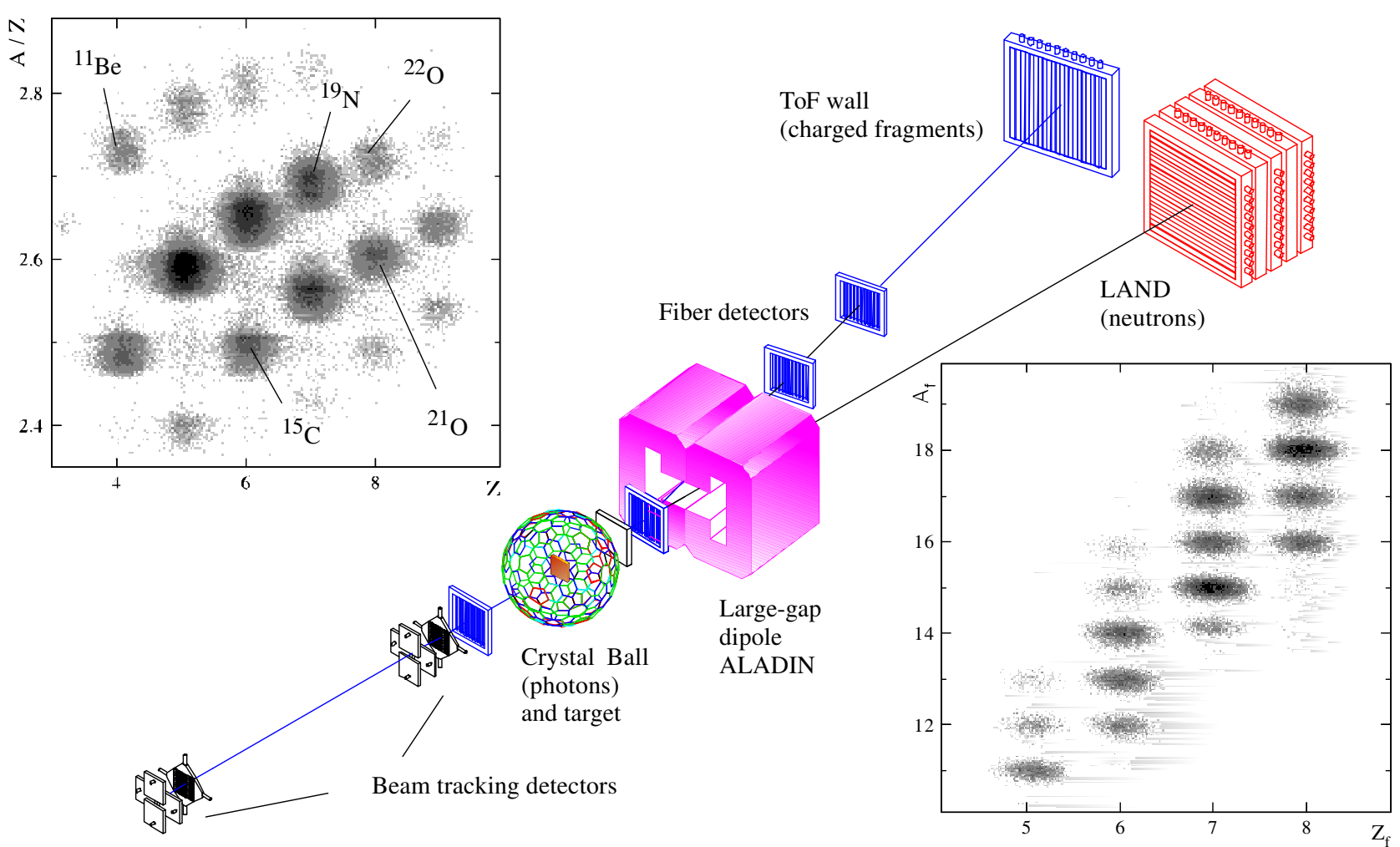

Fig. 2. Schematic drawing of the experimental setup (not to scale) used at GSI for invariant-mass spectroscopy of exotic nuclei. Incoming beam ions are tracked and identified on an event-by-event basis (upper left frame). The outgoing fragments after the target are deflected by the large-gap dipole ALADIN and identified in mass and charge through various position-, time-of-flight-, and energy-loss-measurements (lower right frame). Neutrons are detected with large acceptance of $\pm 80 \mathrm{mrad}$ with the LAND detector located typically $12 \mathrm{~m}$ downstream of the target. Photons emitted by excited fragments are detected by a $4 \pi$ NaI scintillator array (160 NaI crystals) which surrounds the target (Crystal Ball). The kinematically complete measurement allows reconstruction of the excitation energy utilizing the invariant-mass method. The figure is taken from ref. [19].

by (multiple) photon emission. The above relation can be rewritten as

$$
M=\sqrt{\sum_{j} m_{j}^{2}+\sum_{j \neq k} \gamma_{j} \gamma_{k} m_{j} m_{k}\left(1-\beta_{j} \beta_{k} \cos \theta_{j k}\right)}+E_{F}
$$

in terms of ground-state masses $m$, velocities $\beta$, and relative angles $\theta_{j k}$ between neutrons, and neutrons and fragment. The residual excitation energy $E_{F}$ of the heavy fragment can be determined by evaluating the sum energy $E_{\gamma}^{\text {sum }}$ of all emitted $\gamma$ rays in the rest frame of the projectile. This requires high efficiency since the decay might in general involve $\gamma$ cascades, i.e., a calorimetric measurements is needed.

With the setup shown in fig. 2, the excitation energy can be deduced with a resolution of around $200 \mathrm{keV}$ close to the threshold and around $2 \mathrm{MeV}$ in the GDR region $(15 \mathrm{MeV})$. The resolution is dominated by the neutron measurement and the determination of $E_{\gamma}^{\text {sum }}$. The latter measurement introduces in addition a non-trivial energy response of the apparatus caused by an incomplete detection of the $\gamma$ sum energy. Since correcting for this effect involves assumptions on the decay pattern in the simulation, the calorimetric property of the detector is of utmost importance to avoid model dependencies in the analysis. The distorted spectrum causes also limitations in the extraction of the dipole polarizabilty, which is obtained from inverse energy-weighted integral of the $B(E 1)$ strength distribution extracted from the Coulomb excitation cross section. To cope with that difficulty, a new method has been developed recently by Rossi et al. [20] to de-convolute the measured spectrum before integration. This will be discussed later in sects. 3.2 and 4 .

\section{Dipole response of neutron-rich nuclei}

\subsection{Halo nuclei}

Halo nuclei exhibit a very characteristic dipole response which is directly related to the far extending neutron density. As an example, we discuss the one-neutron halo nucleus ${ }^{11} \mathrm{Be}$, which has a neutron separation energy $S_{n}=$ $0.5 \mathrm{MeV}$, while for ${ }^{10} \mathrm{Be} S_{n}=6.8 \mathrm{MeV}$. The structure of ${ }^{11} \mathrm{Be}$ can thus be well approximated by a single-particle model, where the last neutron is bound loosely to the ${ }^{10} \mathrm{Be}$ core. In contrast to the normal shell ordering, the groundstate spin of ${ }^{11} \mathrm{Be}$ is found to be $J^{\pi}=1 / 2^{+}$while the first excited state at $320 \mathrm{keV}$ is the $p$-state with $J^{\pi}=1 / 2^{-}$. 


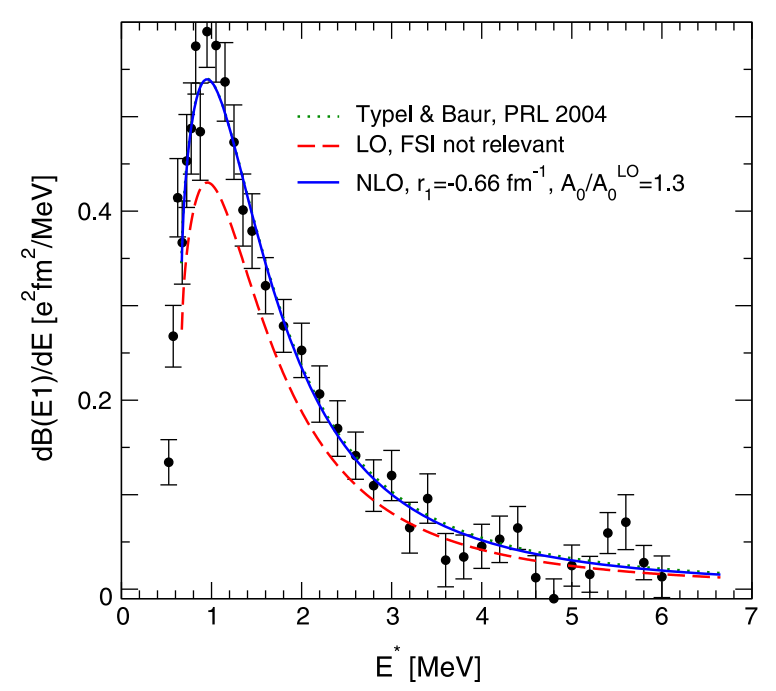

Fig. 3. Dipole-strength distribution of ${ }^{11} \mathrm{Be}$ extracted from the cross section for Coulomb dissociation $\left({ }^{11} \mathrm{Be} \rightarrow{ }^{10} \mathrm{Be}+n\right)$ at $520 \mathrm{MeV} / \mathrm{u}$ [25]. The curves show theoretical calculations of Hammer and Phillipsen in leading (dashed) and next-toleading (solid) order using the Halo-EFT approach [28]. The dotted curve coinciding with the solid curve displays a theoretical analysis by Typel and Baur in an effective-range approach scaled with a spectroscopic factor of 0.7 [30]. The figure is taken from ref. [28].

This so called "parity inversion" is explained theoretically, for instance, on the basis of a particle-rotational-coupling model in ref. [21] or a particle-vibration-coupling model in refs. $[22,23]$. The main component of the neutron singleparticle wave function in the ${ }^{11} \mathrm{Be}$ ground state is the $\left.{ }^{10} \mathrm{Be}\left(0^{+}\right) \otimes \nu 1 s_{1 / 2}\right\rangle$ configuration with a probability of around 70 to $80 \%$ [24] and a rms radius of 5.7(4) fm [25]. With a probability of $\approx 20 \%$, the neutron couples in a $d$-wave $\left|{ }^{10} \operatorname{Be}\left(2^{+}\right) \otimes \nu 0 d_{5 / 2}\right\rangle$ to the first excited $2^{+}$state of ${ }^{10} \mathrm{Be}$ at $3.4 \mathrm{MeV}$.

Several experiments have been performed to measure the differential Coulomb breakup cross section for ${ }^{11} \mathrm{Be}[25-27]$. An extremely large cross section has been found peaking at very low excitation energy of around $600 \mathrm{keV}$ above the separation threshold. Figure 3 shows the $B(E 1)$ distribution extracted from the experiment of ref. [25]. A similar setup as discussed in sect. 2 has been used. The $B(E 1)$ distribution has been extracted from the cross section after subtracting nuclear contributions and the cross section in coincidence with $\gamma$-rays, i.e., the component yielding ${ }^{10} \mathrm{Be}$ in its first excited $2^{+}$ state. The $B(E 1)$ distribution reflects thus the groundstate transitions only, i.e., the breakup related to the $\left.\left.\right|^{10} \mathrm{Be}\left(0^{+}\right) \otimes \nu 2 s_{1 / 2}\right\rangle$ halo configuration.

Figure 3 shows a comparison of the experimentally determined $B(E 1)$ distribution to a theoretical calculation based on halo effective field theory (EFT) [28]. The characteristic shape of the spectrum is very well reproduced by the theory. The normalization is a free parameter in the theory since not all coupling constants are fixed in the next-to-leading order calculation. With the free parameter adjusted to the $B(E 1)$ from the Coulomb breakup data, the theory predicts a charge rms radius of $2.42 \mathrm{fm}$ and a neutron rms radius of 5.6(6) fm. These values are in very good agreement with the optical measurement for the charge radius by Nörtershäuser et al. of $2.463(16) \mathrm{fm}$ [29], and the neutron $\mathrm{rms}$ radii of $5.7(4) \mathrm{fm}$ [25] and 5.77(16) fm [27] extracted from Coulomb breakup data using a single-particle model. The dotted curve coinciding with the solid curve (representing the EFT result) displays a theoretical analysis by Typel and Baur in an effective-range approach, where the normalization of the neutron single-particle wave function has been scaled to fit the data corresponding to a spectroscopic factor of 0.7 [30].

The large dipole transition probability observed in Coulomb breakup experiments is directly related to the spatial extension of the halo wave function, as already predicted in the famous work of Hansen and Jonson [5] when interpreting the discovery experiment of Tanihata et al. [4], and coining the name neutron halo. If we consider a one-neutron halo nucleus in the single-particle core+neutron picture sketched above, and if we consider dipole transitions leading to a final state $\left|\phi_{f}(\mathbf{r}, \mathbf{q})\right\rangle$ of the core in its ground state plus a neutron with relative momentum q, we expect a dipole-strength distribution of the form

$$
\frac{\mathrm{d} B(E 1)}{\mathrm{d} E_{\mathrm{rel}}}=\left|\left\langle\phi_{f}(\mathbf{r}, \mathbf{q})\left|(Z e / A) r Y^{1}\right| \phi(\mathbf{r})\right\rangle\right|^{2},
$$

where $E_{\text {rel }}$ is the relative energy between neutron and core in the final state. If we further consider for simplicity plane waves for the final state $\left|\phi_{f}(\mathbf{r}, \mathbf{q})\right\rangle$ and neglect final-state interaction, eq. (4) has the form of a Fourier transform of the single-particle wave function $\phi$ weighted through the dipole operator with the relative coordinate $r$. The large radial extension of the halo wave function thus translates into large $E 1$ transition probabilities for small $q$ (small $\left.E_{\text {rel }}\right)$ in the final state.

A similar effect is present for the $1^{-}$transition to the first excited and only bound state of ${ }^{11} \mathrm{Be}$ at $320 \mathrm{keV}$. Millener et al. measured a lifetime of the $1 / 2^{-}$state of only $166(15)$ fs [31], which is the fastest known $E 1$ transition between bound states. As pointed out by Hansen et al. [32], this is a clear indication for the halo character of both $1 / 2^{-}$and $1 / 2^{+}$states of ${ }^{11} \mathrm{Be}$ due to the very same reason as explained above for the continuum transition. The situation is illustrated in fig. 4, where the radial wave functions of the two states are shown. Both wave functions exhibit a similar long tail caused by the weak binding. The somewhat faster decay of the $p$-wave function despite even lower binding is due to the centrifugal barrier not present for the $s$ state. The contribution to the overlap integral between the two states becomes huge for large values of $r$, enhanced by the $r$ weighting for the dipole transition. Nakamura et al. [33] measured the Coulomb excitation cross section to the first excited state. The deduced $B(E 1)$ value is in good agreement with the life-time measurement mentioned above. 


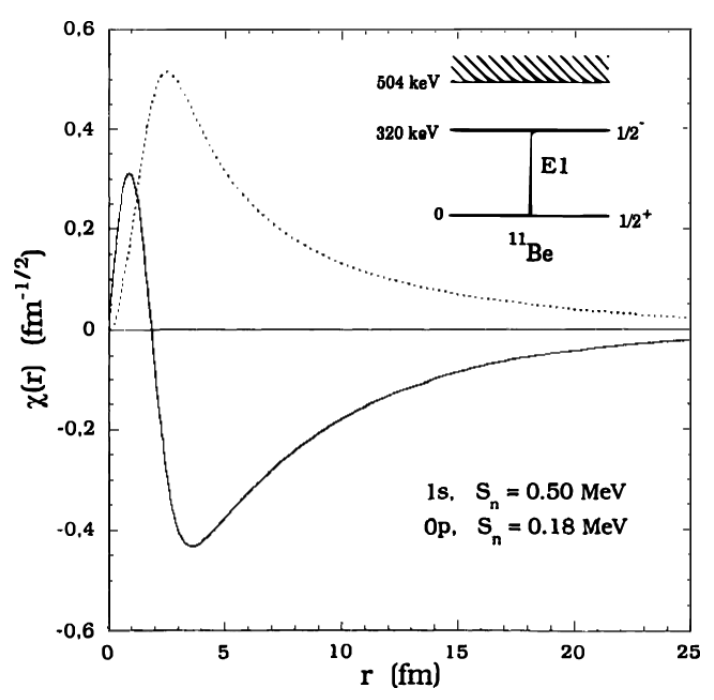

Fig. 4. Radial wave function $\chi(r)=r R(r)$ for the two bound $s$ (solid curve) and $p$ (dotted curve) states of ${ }^{11}$ Be corresponding to the states indicated in the level scheme (inset). The respective $\mathrm{rms}$ radii are 6.0 and $5.7 \mathrm{fm}$, while the core radius is $2.5 \mathrm{fm}$. The figure is taken from the review of Hansen, Jensen, and Jonson [32].

The huge sensitivity of Coulomb breakup to the extended tail of the wave function makes it a very useful tool to study the halo property of nuclei. The large cross section allows experiments even with low beam intensities, i.e., for the most exotic nuclei produced at the fragmentation facilities. The method has been applied to other halo nuclei like ${ }^{15} \mathrm{C}$ and ${ }^{19} \mathrm{C}$ as well. A recent experiment extended the halo search to heavier loosely-bound nuclei. The large cross section observed in an inclusive Coulomb-breakup measurement of ${ }^{31} \mathrm{Ne}$ clearly indicated the halo character of ${ }^{31} \mathrm{Ne}$ [34]. From a comparison of the measurement with predictions by the breakup model discussed above, Nakamura et al. [34] could conclude that the ground-state wave function has a strong $p$-wave intruder component which causes in combination with the low binding energy the halo of ${ }^{31} \mathrm{Ne}$.

For two-neutron halo nuclei, the situation becomes more complex because the $E 1$ response will also reflect correlations among the neutrons in the initial as well as in the final state. Since the two neutrons are bound only weakly to the $A-2$ core, a description of two-neutron halo nuclei in three-body models seems adequate. A particular clear example is the borromean nucleus ${ }^{6} \mathrm{He}$, where the two neutrons are bound by around $1 \mathrm{MeV}$ to the $\alpha$ core with a particle separation energy and first excited state at around $20 \mathrm{MeV}$. The $\alpha$ particle can thus be considered as a good inert core. Moreover, the $\alpha-n$ and $n-n$ subsystems are unbound. This is the case also for the borromean nucleus ${ }^{11} \mathrm{Li}$. Although, the approximation of an inert core might be less good since ${ }^{9} \mathrm{Li}$ has the first excited state at $2.7 \mathrm{MeV}$. However, ${ }^{11} \mathrm{Li}$ is even less bound with $S_{2 n}=0.4 \mathrm{MeV}$ while the core has a particle separation threshold of $S_{n}=4.1 \mathrm{MeV}$. For both nuclei, the low-lying dipole strength has been studied experimentally in exclusive Coulomb-dissociation experiments $[35,36]$.

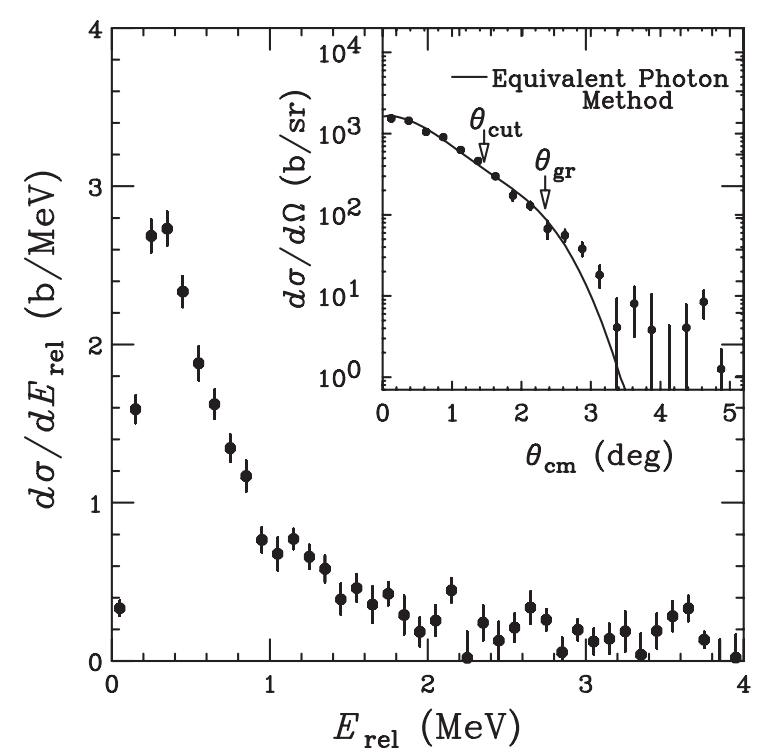

Fig. 5. Excitation cross section for the two-neutron halo nucleus ${ }^{11} \mathrm{Li}$. The data were taken with a $70 \mathrm{MeV} / \mathrm{u}$ beam on a lead target for small scattering angles below $\theta_{\text {cut }}$ (see inset) [36]. The cross section is thus Coulomb dominated reflecting dominantly $E 1$ transitions to the continuum. The figure is taken from ref. [36].

The differential cross section for Coulomb breakup measured at RIKEN for ${ }^{11} \mathrm{Li}[36]$ is shown in fig. 5. The cross section exhibits a very pronounced peak at very low relative energy $E_{\text {rel }}$ of around $0.35 \mathrm{MeV}$ with a cross section of almost $0.3 \mathrm{~b} / \mathrm{MeV}$. This huge cross section constitutes the largest $E 1$ transition strength observed in nuclei so far [36] reflecting the pronounced halo structure of ${ }^{11} \mathrm{Li}$. Besides the very large spatial extension of the halo, the strength is further enhanced due to $n-n$ correlations in the ${ }^{11} \mathrm{Li}$ ground-state wave function (see discussion below).

The peak position at around $0.35 \mathrm{MeV}$ in relative energy corresponds to an excitation energy $E \approx 0.75 \mathrm{MeV}$. This is in remarkable good agreement with the theoretical work of Barranco and Bortignon et al. [37] predicting a strong $E 1$ transition to a state located at $0.75 \mathrm{MeV}$. It should be noted, that the same theory correctly reproduces the experimentally found [38] strong mixing of $s$ and $p$-wave configurations in the ${ }^{11} \mathrm{Li}$ ground state with only very little $d$ admixture.

A low-lying $E 1$ resonance in ${ }^{11} \mathrm{Li}$ has also been reported from a recent experiment performed at TRIUMF utilising a very different reaction, namely proton inelastic scattering [39]. The spectrum is shown in fig. 6. A resonance energy of $0.80(2) \mathrm{MeV}$ was extracted, very close to the energy seen in the $B(E 1)$ distribution extracted from the Coulomb-excitation experiment discussed above. This is an important experimental finding, since it corroborates the resonance character of the $E 1$ transition strength observed in Coulomb excitation. An explanation of the large Coulomb cross section peaking at the same energy but resulting from non-resonant direct breakup as discussed above for ${ }^{11} \mathrm{Be}$ can be excluded due to the 


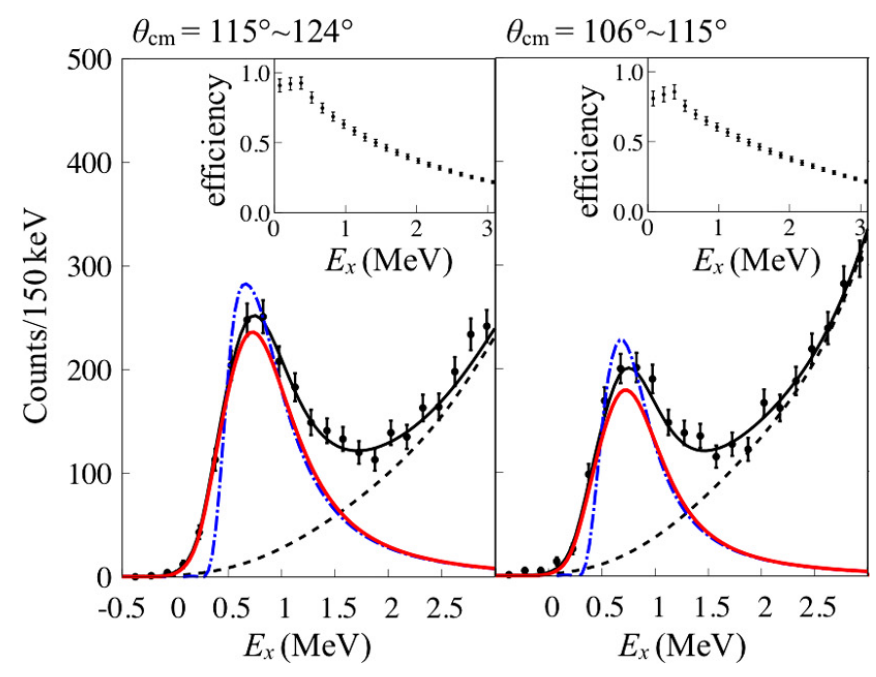

Fig. 6. Excitation spectrum of the two-neutron halo nucleus ${ }^{11} \mathrm{Li}$ resulting from nuclear excitation in a $\left(p, p^{\prime}\right)$ scattering at $6 \mathrm{MeV} / \mathrm{u}$ and different angular ranges as indicated in the figure [39]. The angular distribution of the resonance-like structure indicates a dipole transition at around $0.8 \mathrm{MeV}$. The figure is taken from ref. [39].

strong final-state interaction in the presence of a resonance. We cannot follow the arguments of Tanaka et al. that the resonance seen in $\left(p, p^{\prime}\right)$ is excited most likely by an isoscalar transition. Firstly, a dipole resonance corresponding to a vibration of the halo neutrons versus the core should be excited strongly by isovector transitions. Secondly, even an isoscalar probe would excite such a resonance much stronger by an isovector transition compared to an isoscalar transition since the probing particle interacts much stronger with the neutrons due to the peripheral nature of the scattering process. We rather conclude here that the two experiments see the same state excited by a $l=1$ transition, which corresponds to the theoretically predicted soft dipole resonance, i.e., a vibration of the two-neutron halo against the core.

In a soft $E 1$ excitation of two-neutron halo nuclei, only the relative motion of the core and the two neutrons will be relevant. By separating the strength of the dipole motion into that of the core, that of the halo nucleons, and that of the relative motion between core and halo neutrons one obtains a "cluster" sum rule for the $E 1$ strength. This sum rule then involves the corresponding mean-square distances among the clusters, like the average $n-n$ distance or the relative mean-square distance $\left\langle r_{\mathrm{c}, 2 n}\right\rangle$ between the core and the centre-of-mass of the two neutrons. $\left\langle r_{\mathrm{c}, 2 n}\right\rangle$, and thus the low-lying dipole strength will depend sensitively on spatial correlations among the two neutrons. In terms of $\left\langle r_{\mathrm{c}, 2 n}\right\rangle$, the non-energy weighted cluster sum rule can be expressed as follows [40]:

$$
B(E 1)=\frac{3}{4 \pi}\left(\frac{Z e}{A}\right)^{2} 4\left\langle r_{\mathrm{c}, 2 n}\right\rangle .
$$

The extracted $B(E 1)$ distribution from the differential cross section for Coulomb dissociation of ${ }^{6} \mathrm{He}$ at
$240 \mathrm{MeV} / \mathrm{u}$ has been analyzed in that way. From the integrated $E 1$ strength up to $10 \mathrm{MeV}$ of $1.2(2) e^{2} \mathrm{fm}^{2}$, a root-mean square distance $r_{\alpha, 2 n}=3.36(39) \mathrm{fm}$ between the $\alpha$ core and the two halo neutrons has been deduced in ref. [35], which is in good agreement with three-body models (see the compilation given in table 3 of ref. [41]). The recoil of the $\alpha$-particle associated with the $n-n$ correlations contributes (among other effects) to the chargeradius difference between ${ }^{4} \mathrm{He}$ and ${ }^{6} \mathrm{He}$. Esbensen et al. estimate $\left\langle r_{\alpha, 2 n}\right\rangle=13.8(5) \mathrm{fm}^{2}[40]$ from the measured charge radius of ${ }^{6} \mathrm{He}$ of 2.054(14) fm [42], corresponding to $r_{\alpha, 2 n}=3.71(7) \mathrm{fm}$, which is as well in good agreement with the experimental value from ref. [35].

The charge radius of ${ }^{6} \mathrm{He}$ was also calculated with the Gamow shell model by Papadimitriou et al. [43]. From fig. 4 of ref. [43], we read off a contribution of $1.14 \mathrm{fm}$ to the charge-radius difference between ${ }^{6} \mathrm{He}$ and ${ }^{4} \mathrm{He}$ related to the recoil, if we subtract the two values quadratically. This we can compare to the root-mean square distance of the $\alpha$-particle and the center-of-mass of ${ }^{6} \mathrm{He}$ of 1.12 (13) fm deduced from the dipole strength in ref. [35], showing again good agreement. A more precise determination of the dipole strength would be desirable for a more stringent comparison with theory and other methods, in order to enable conclusions on the importance of spatial $n-n$ correlations in the ${ }^{6} \mathrm{He}$ ground state.

If the same analysis is applied to ${ }^{11} \mathrm{Li}$, based on the up to $3 \mathrm{MeV}$ integrated $B(E 1)$ strength of $1.78(22) \mathrm{fm}^{2}$ extracted from the Coulomb-dissociation cross section measurement discussed above, a value $r_{c, 2 n}=5.01(32) \mathrm{fm}$ is deduced [36]. Esbensen et al. extracted a value of $r_{c, 2 n}=5.97(22) \mathrm{fm}[40]$ based on the charge radii of ${ }^{11} \mathrm{Li}$ and ${ }^{9} \mathrm{Li}$. It was discussed in ref. [40], that this $1.5 \sigma$ discrepancy could well be the result of core polarization, neglected in the 3-body model. It was also pointed out in ref. [40], that the effect of core polarization is expected to be much smaller for the $\alpha$-core (for the reasons discussed above). So a precise measurement of the $B(E 1)$ strength for ${ }^{6} \mathrm{He}$ would be desirable to check the consistency of the interpretation of charge radii and Coulomb breakup. Since the change of the core size in a halo nucleus compared to the free core nucleus affects the charge radius but not the dipole strength, the discussed difference could provide direct evidence for the core swelling in ${ }^{11} \mathrm{Li}$, if the method of comparison could be benchmarked. A recent experiment performed at RIBF [44] is expected to extract the dipole strength of ${ }^{6} \mathrm{He}$ for a large energy region and with high statistics and improved resolution. It is expected that the precision of the extracted radius $r_{c, 2 n}$ for the ${ }^{6} \mathrm{He}$ halo using above method will be significantly smaller than the difference found for ${ }^{11} \mathrm{Li}$ as discussed above, which will provide a precise check of the consistency of the two methods.

\subsection{Giant and pygmy dipole resonances in neutron-rich nuclei}

The dipole strength distribution of neutron-rich light nuclei, in particular the halo nuclei discussed above, has been measured so far only in the low-energy region related to 


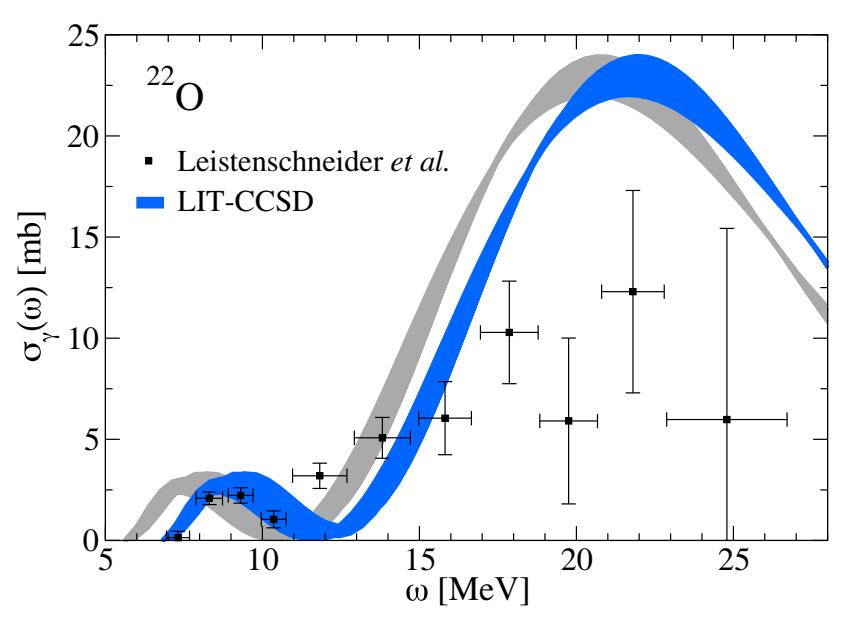

Fig. 7. Photo-absorption cross section of ${ }^{22} \mathrm{O}$ calculated in the coupled-cluster approach and using the Lorentz integral transform method. The theoretical curve (grey) has been shifted to the experimental neutron threshold (blue curve) and is compared to the photo-neutron cross section deduced from a measurement of electromagnetic dissociation at $600 \mathrm{MeV} / \mathrm{u}$ [46]. The figure is taken from the work of Bacca et al. [49].

the response of the halo. The same holds for some measurements for heavier nuclei like ${ }^{26} \mathrm{Ne}$ [45], where only the low-energy part of the strength has been covered. The much higher-lying giant resonance could so far never be reached experimentally for a halo nucleus. We would expect well separated regions in the dipole response related to halo excitations and the giant resonance. It would be exciting to compare a giant dipole resonance in a halo nucleus to the one of the free core. Since the GDR is located at rather high energy in light nuclei, such a measurement using electromagnetic excitation requires rather high beam energies as discussed in sect. 2. An attempt in this direction is planned at GSI for ${ }^{6} \mathrm{He}$ at $1 \mathrm{GeV} / \mathrm{u}$ using the $\mathrm{R}^{3} \mathrm{~B}$ setup at GSI.

The experimental studies of the dipole response in a wide-enough energy region to cover both PDR and GDR excitation has been restricted to few nuclei only, so far. These are measurements for the oxygen isotopes [46], ${ }^{68} \mathrm{Ni}[20]$, and few nuclei around ${ }^{132} \mathrm{Sn}[47,48]$. We restrict the discussion here to these results.

The most neutron-rich nucleus reached experimentally in the above mentioned GSI experiment was ${ }^{22} \mathrm{O}$. The photo-neutron cross section extracted from the measurement of the neutron-decay part of the Coulomb dissociation cross section is shown in fig. 7. The experimental technique including the setup and analysis method used is described in sect. 2. The data show significant dipole strength below the GDR region, exhibiting a peak structure at around $9 \mathrm{MeV}$, which could be related to a pygmy resonance appearing in this neutron-rich nucleus. The data are compared to a theoretical ab initio calculation of the photo-absorption cross section of Bacca et al. [49]. After shifting the theoretical distribution to fit the experimental separation energy (blue band), the lowenergy peak at $9 \mathrm{MeV}$ is very well reproduced by the theory. The discrepancy in the GDR region might partly be

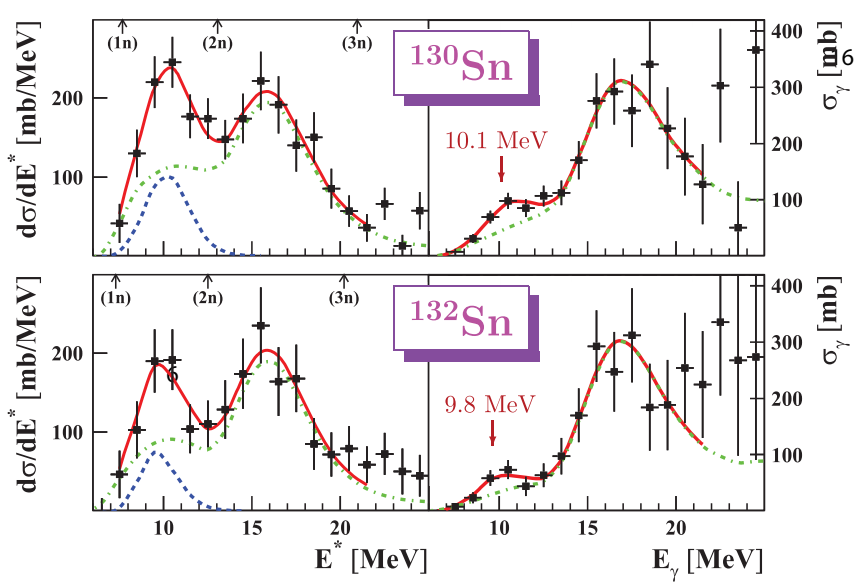

Fig. 8. Dipole response of ${ }^{130,132} \mathrm{Sn}$. The left panels show the differential cross section for electromagnetic excitation and neutron decay of $\mathrm{Sn}$ projectiles at $500 \mathrm{MeV} / \mathrm{u}$ on a $\mathrm{Pb}$ target. Arrows indicate the neutron-separation thresholds. The right frames show the corresponding deduced $(\gamma, n)$ photo-nuclear cross sections $\sigma_{\gamma}$. The spectra have been fitted by a Lorentzian for the GDR (green dash-dotted line) and a Gaussian for the low-energy PDR peak (blue dashed line). The solid line corresponds to the sum of the two components. The figure is taken from Adrich et al. [47].

attributed to the open proton channel not covered by the experiment. But clearly, more precise data are desirable to conclude on the GDR part of the dipole response.

For heavier neutron-rich nuclei, the development of a collective low-energy dipole resonance has been predicted [6] showing the characteristics of a vibration of the excess neutrons against the core, a picture usually presupposed when using the term pygmy dipole resonance. The first experimental evidence providing support for this prediction resulted from a Coulomb excitation experiment performed at GSI using radioactive beams of neutron-rich nuclei around ${ }^{132} \mathrm{Sn}$ at beam energies around $500 \mathrm{MeV} / \mathrm{u}$. The results obtained by Adrich et al. [47] for ${ }^{130} \mathrm{Sn}$ and ${ }^{132} \mathrm{Sn}$ are shown in fig. 8. The left part displays the differential cross section for electromagnetic excitation and neutron decay for up to three neutrons. The right part shows the deduced photo-neutron cross sections from the same data. The pronounced difference in the shape of the two spectra is caused by the virtual-photon spectrum discussed in sect. 2, which enhances the low-energy part of the dipole response.

A peak structure at around $10 \mathrm{MeV}$ is visible for both nuclei, close to the theoretical prediction of the PDR [6]. The solid curves show fits to the experimental cross sections assuming a Lorentzian shape for the GDR, and a Gaussian shape for the low-energy peak. The experimental response resulting from a complete simulation of the experiment, including the simulation of the decay pattern, is taken into account in the fitting procedure. The resulting strength for the low-energy pygmy peak amounts to $5(2) \%$ and $3(2) \%$ of the energy-weighted dipole sum rule for the two isotopes ${ }^{130} \mathrm{Sn}$ and ${ }^{132} \mathrm{Sn}$, respectively, which is also in good agreement with the theoretical relativistic mean field prediction. 


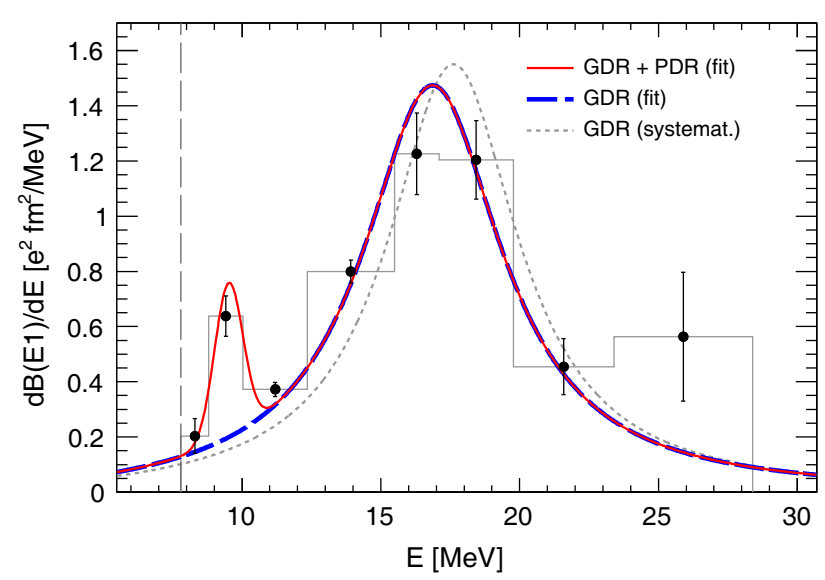

Fig. 9. E1 strength distribution above neutron threshold deduced from the electromagnetic dissociation cross section for ${ }^{68} \mathrm{Ni}$ measured at $503 \mathrm{MeV} / \mathrm{u}$ with a $\mathrm{Pb}$ target. The curves show fits to the data and a Lorentzian with parameters from the GDR systematics for stable nuclei for comparison. The figure is taken from ref. [20].

The relatively large uncertainty is dominated by the experimental response as discussed in sect. 2. The main contribution to this response stems from an incomplete detection of the residual excitation energy of the fragment, which is determined in the experiment by the total energy released by $\gamma$-rays detected in the Crystal Ball detector (see sect. 2). Incomplete detection of this energy due to inefficiency will shift strength towards lower excitation energy. The effect is displayed in fig. 8 by the green dash-dotted curve, which exhibits a shoulder at low energy resulting from the simulation of the Lorentziandistributed strength for the GDR. This causes a correlation of the fit parameters for the PDR and GDR strength, resulting in the relatively large uncertainty. Since this systematic uncertainty is close to be the same for the two measurements, an averaging of the sum-rule exhaustion for the two isotopes will not reduce the experimental uncertainty. Clearly, improvements on the experimental apparatus are called for. The future $\mathrm{R}^{3} \mathrm{~B}$ setup will include a new calorimeter for photon detection with high granularity and better resolution. The high granularity will besides enabling a more precise Doppler correction also allow for a better suppression of background in the $\gamma$ detector originating from Bremsstrahlung produced by $\delta$-electrons.

For the analysis of the Coulomb excitation of ${ }^{68} \mathrm{Ni}$, a new procedure has been developed by Rossi et al. [20] to "unfold" the measured spectrum taking into account the above discussed response of the apparatus. Here, the fitting parameters are the cross sections in pre-defined energy bins. The fit is performed to best describe simultaneously the measured quantities like neutron-energy spectra, detected $\gamma$ energy, etc., and their correlations. The result is shown in fig. 9 by the filled circles. The error bars include now the uncertainties introduced by correlations between different energy regions caused by the response discussed above, and are thus not only of statistical nature. The widths of the bins is adjusted to be larger than the overall resolution to avoid additional correlations of

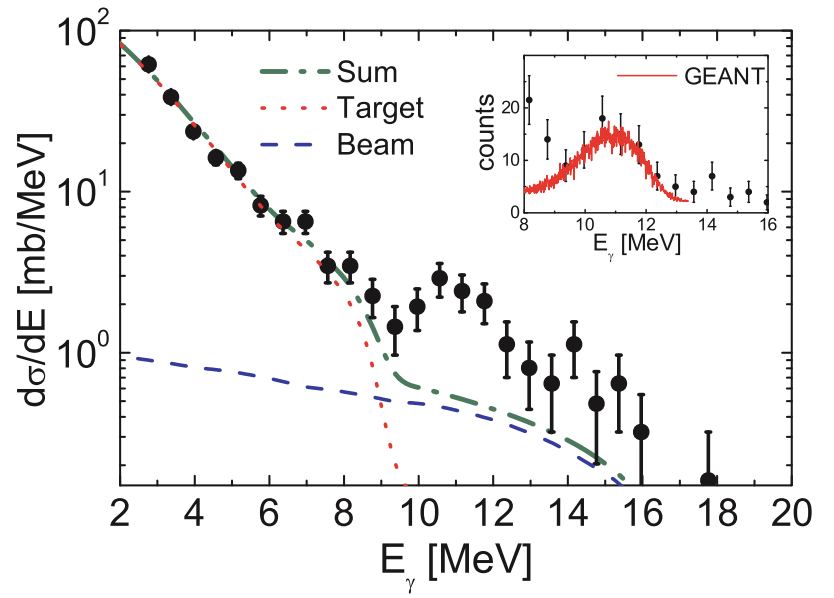

Fig. 10. Cross section for $\gamma$ decay after Coulomb excitation of ${ }^{68} \mathrm{Ni}$ at $600 \mathrm{MeV} / \mathrm{u}$ on a $\mathrm{Au}$ target. The curves represent statistical-model calculations for target and projectile excitation. The inset shows a comparison of the data with a GEANT simulation for a $\gamma$-transition at $11 \mathrm{MeV}$. The figure is taken from ref. [50].

neighbouring energy intervals in order to stabilize the fit. (This results in a varying bin width related to the resolution at the corresponding energy.) The advantage of this procedure is that the extracted spectrum is effectively unfolded from the instrumental response, and does not need a pre-defined shape of the fit function. The resolution is included in the bin width. A direct comparison with theory is thus much better possible.

The experimental result for the $B(E 1)$ strength distribution extracted using above described procedure is compared in fig. 9 with a Lorentzian-shaped strength distribution adopting parameters from the systematics for stable nuclei (dotted curve), which shows good agreement with the data. A free fit of a Lorentz curve (blue dashed curve), however, results in a peak energy slightly below the systematics for stable nuclei. A more systematic experimental investigation is called for to clarify if the GDR in neutron-rich nuclei exhibits significant differences compared to stable nuclei.

The dipole-strength distribution deduced experimentally for ${ }^{68} \mathrm{Ni}$ as displayed in fig. 9, exhibits a cross section enhancement at around $9 \mathrm{MeV}$ excitation energy, which is identified as the PDR. A fit to the data (red solid curve) results in a position at $9.55(17) \mathrm{MeV}$ and a strength exhausting $2.8(5) \%$ of the energy-weighted dipole sum rule. Another experiment performed by Wieland et al. [50] measured the $\gamma$ decay after Coulomb excitation of ${ }^{68} \mathrm{Ni}$ at a beam energy of $600 \mathrm{MeV} / \mathrm{u}$. The spectrum displayed in fig. 10 exhibits a clear peak structure at $11 \mathrm{MeV}$, significantly higher than observed in the neutron decay. The reason for this discrepancy is not understood. A high-statistics experiment preferentially measuring both neutron and $\gamma$ decay in the same experiment would be desirable to illuminate this puzzle. The method of virtual-photon scattering has been recently applied also to a measurement of ${ }^{70} \mathrm{Ni}[51]$, showing an enhancement of $E 1$ strength around $10.5 \mathrm{MeV}$. 


\section{Dipole response and the symmetry energy}

The nuclear symmetry energy $S(\rho)$ describes the difference between the equation of state (EoS) for symmetric nuclear matter and for neutron matter as a function of density $\rho=\rho_{p}+\rho_{n}$. Properties of nuclei are governed by the EoS around saturation density $\rho_{0}$ including the dependence on isospin asymmetry $\delta=\frac{\rho_{n}-\rho_{p}}{\rho_{n}+\rho_{p}}$. To characterise the properties of the EoS with few parameters an expansion around $\rho_{0}$ and asymmetry $\delta$ is useful:

$$
E(\rho, \delta)=E(\rho, 0)+S(\rho) \delta^{2}+O\left[\delta^{4}\right],
$$

where $E(\rho, 0)$ describes the EoS of symmetric matter, and the symmetry energy $\left.S(\rho) \equiv \frac{\partial E(\rho, \delta)}{\partial \delta}\right|_{\delta=0}$ the dependence on asymmetry, which are expanded around $\rho_{0}$ :

$$
\begin{aligned}
E(\rho, 0)= & E\left(\rho_{0}, 0\right)+\frac{1}{2} K_{0}\left(\frac{\rho-\rho_{0}}{3 \rho_{0}}\right)^{2} \\
& +O\left[\left(\rho-\rho_{0}\right)^{3}\right], \\
S(\rho, 0)= & J+L \frac{\rho-\rho_{0}}{3 \rho_{0}}+\frac{1}{2} K_{\mathrm{sym}}\left(\frac{\rho-\rho_{0}}{3 \rho_{0}}\right)^{2} \\
& +O\left[\left(\rho-\rho_{0}\right)^{3}\right],
\end{aligned}
$$

where $\left.K_{0} \equiv 9 \rho_{0}^{2} \frac{\partial^{2} E(\rho, 0)}{\partial \rho^{2}}\right|_{\rho=\rho_{0}}$ gives the incompressibility of symmetric nuclear matter and $\left.K_{\text {sym }} \equiv 9 \rho_{0}^{2} \frac{\partial^{2} S(\rho)}{\partial \rho^{2}}\right|_{\rho=\rho_{0}}$ the incompressibility of the symmetry energy. $J$ is defined as the symmetry energy at saturation density, and $L$ corresponds to its slope $\left.L \equiv 3 \rho_{0} \frac{\partial S(\rho)}{\partial \rho}\right|_{\rho=\rho_{0}}$ at saturation density.

The parameters of the EoS can be experimentally constraint by bulk properties of heavier nuclei, where the surface terms are less important. In order to describe properties of nuclei and nuclear matter on the same footing, energy density functionals (EDF) derived from selfconsistent mean-field models (SCMF) are utilized. The parameters of the different effective interactions used in the different SCMF models are determined by a fit to properties of nuclei. Most interactions are derived from a fit to masses and radii, which provides already constraints to the EoS parameters. Further constraints are provided, e.g., by also taking into account properties of the collective response like the excitation frequency of the giant monopole resonance, from which the incompressibility $K_{0}$ has been inferred [12]. An elaborated overview and discussion of the connection between nuclear observables and the EoS can be found in the recent review of Roca-Maza and Paar [12].

Modern EDF theories are able to describe nuclear properties of stable nuclei rather accurately. However, the different underlying EDFs still may result in rather different parameters $J$ and $L$ describing the asymmetry dependence of the EoS. Other observables, not used for deriving the EDF, are needed to provide complementary constraints. Two in this respect very promising properties are the neutron-skin thickness $\Delta r_{n p}$ and the dipole polarizability $\alpha_{D}$ of heavy neutron-rich nuclei $[12,52]$. This conclusion is drawn by comparing the different predicted values for $\Delta r_{n p}$ and $\alpha_{D}$ from different EDFs with the corresponding symmetry-energy parameters $J$ and $L$ from the same theories. For both observables, correlations with $J$ and $L$ are found, implying that an accurate measurement of these quantities will provide constraints on $J$ and/or $L$. To determine such properties experimentally for neutronrich exotic nuclei is of particular importance, because the changes of these quantities with respect to symmetric nuclei is most pronounced, and which would allow a systematic study as a function of neutron excess.

The dipole polarizability $\alpha_{D}$ can be obtained from the inverse energy-weighted sum of the dipole strength, and thus from the $1 / E^{2}$-weighted integral of the photoabsorption cross section $\sigma_{\gamma}$, where $E$ refers to the photon energy or excitation energy of the nucleus:

$$
\alpha_{D}=\frac{\hbar c}{2 \pi^{2}} \int_{0}^{\infty} \frac{\sigma_{\gamma}(E)}{E^{2}} \mathrm{~d} E .
$$

The integral in eq. (9) is evaluated with a typical upper value of $30 \mathrm{MeV}$. This covers the energy region relevant for collective excitations. At higher excitation energies, the photo-absorption cross section is dominated by $n-p$ correlations, the so-called "quasi-deuteron" effect. This process is not taken into account in the theoretical calculations of the response, and thus should not be included in the experimental determination for the comparison we discuss here. Small contributions from GDR excitation extending in that region can be approximated by extrapolating the Lorentzian-shaped GDR. However, the experimental determination of $\alpha_{D}$ requires the measurement of the full response below and above threshold up to around $30 \mathrm{MeV}$ excitation energy.

The first and so far only measurement of $\alpha_{D}$ for an unstable neutron-rich nucleus is based on the Coulombexcitation measurement of ${ }^{68} \mathrm{Ni}$ from GSI [20] discussed in the previous section. Figure 11 shows the inverse-energy weighted dipole strength derived from the experimental data (black dots with error bars) compared to theoretical predictions by Piekarewicz [53] based on a relativistic mean field (RMF) model. The different curves result from three different RMF calculations using FSUGold effective interactions with different symmetry-energy slope $L$. This results in different predictions for the neutron-skin thickness of ${ }^{68} \mathrm{Ni}$ as indicated in the figure. But also the predicted dipole response changes dramatically. While the neutron-skin thickness increases for larger $L$ values, the dipole response becomes softer, leading to larger values of the dipole polarizability. This can be seen in the inset, where the cumulated sum of the experimental dipole polarizability is compared to the corresponding RMF calculations.

The experimental result for the dipole polarizability for ${ }^{68} \mathrm{Ni}$ is $\alpha_{D}=3.40(23) \mathrm{fm}^{3}$ [20], when integrating over the data points. This value is corrected by extrapolating to the not covered excitation-energy regions below threshold and above $28 \mathrm{MeV}$ (see fig. 11) by using a Lorentzianshaped distribution with parameters adjusted to the data resulting in $\alpha_{D}=3.9(3) \mathrm{fm}^{3}$, taking into account a conservative estimate of the systematic uncertainty introduced by the extrapolation. This value together with other measurements was used by Roca-Maza et al. [54] to derive 


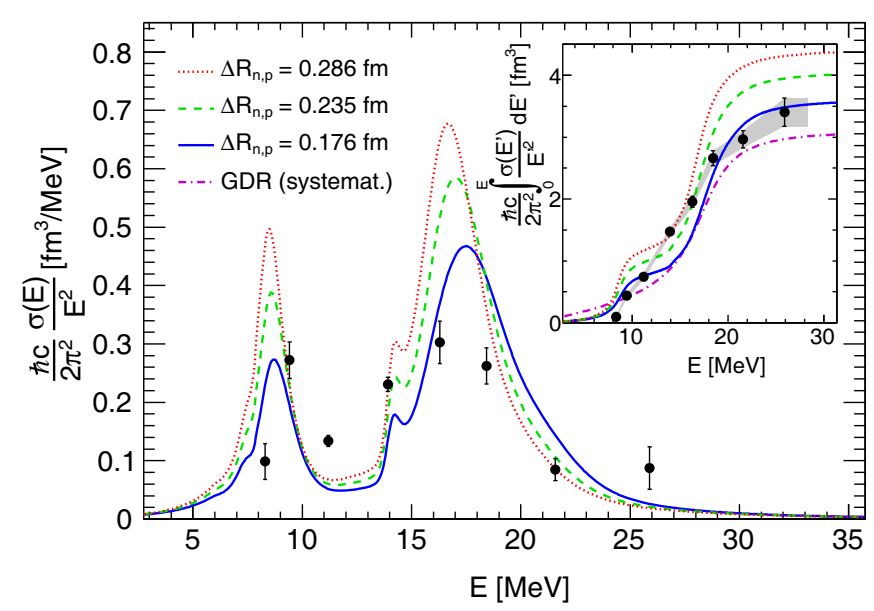

Fig. 11. Left: Inverse energy-weighted dipole strength for ${ }^{68} \mathrm{Ni}$ obtained experimentally (black dots) and theoretically using three different FSUGold interactions with different slope parameters $L$ for the symmetry energy [53] (curves). The inset shows the cumulated sum of the dipole polarizability. The figure is taken from ref. [20].

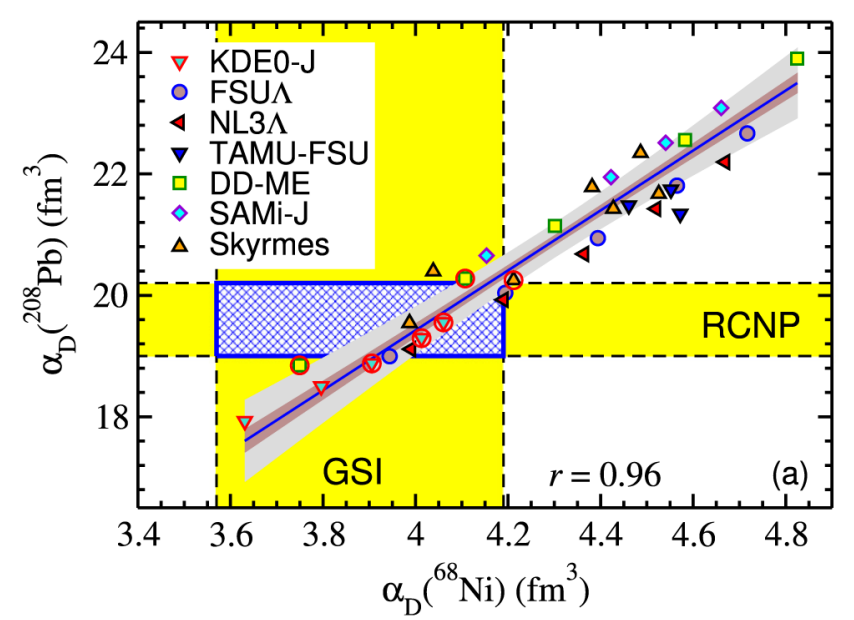

Fig. 12. Correlation of the dipole polarizabity $\alpha_{D}$ of ${ }^{68} \mathrm{Ni}$ with $\alpha_{D}$ of ${ }^{208} \mathrm{~Pb}$ calculated with different density functionals (symbols). The yellow bands indicate the experimental values for the two nuclei. The figure is taken from ref. [54].

constraints on the symmetry-energy parameters. Figure 12 shows the correlation of calculated values of $\alpha_{D}$ for ${ }^{68} \mathrm{Ni}$ and ${ }^{208} \mathrm{~Pb}$ using many different DFTs with correspondingly different values for $J$ and $L$. The yellow bands indicate the experimental values for the two nuclei. The experimental value for ${ }^{208} \mathrm{~Pb}$ was obtained by an analysis of Tamii et al. using the world data on photo-absorption including their new data obtained from a $\left(p, p^{\prime}\right)$ Coulombexcitation measurement [55]. Both data overlap with the theoretical calculation and select a range of DFTs predicting $\alpha_{D}$ correctly for both nuclei. Also the third data point for ${ }^{120} \mathrm{Sn}[56]$ included in this analysis provides a consistent selection, from which a range of allowed values of $J=30-35 \mathrm{MeV}$, and $L=20-66 \mathrm{MeV}$ was derived [54].
A similar analysis performed in refs. [48, 57] based on only the low-energy pygmy peak in the spectrum arrived at somewhat tighter constraints. The restriction of such an analysis to only part of the dipole strength, however, makes the approach rather model dependent. The definition of pygmy strength in experiment and theory which are compared, for instance, might not be unequivocal. It has been pointed out by Reinhard and Nazarewicz [11] that a better correlated and more robust observable would be the polarizability of the nucleus.

The allowed values for $J$ and $L$ after taking into account the experimental constraints cover still a rather wide range. More precise data on different observables are demanded to come closer to the theoretical limit inherent to the method of $\pm 10 \mathrm{MeV}$ for $L$, given by the scatter of theoretical predictions in the correlation analysis [12]. The experimental uncertainty for the determination of $\alpha_{D}$ using Coulomb excitation of radioactive beams are dominated by the effects due to the experimental response as discussed in the previous section. For measurements at the future $\mathrm{R}^{3} \mathrm{~B}$ setup, an accuracy of $5 \%$ for the determination of $\alpha_{D}$ is anticipated. The measurement of the neutron-skin thickness for neutron-rich unstable nuclei is challenging. A promising new method has been recently proposed [58], which is based on a measurement of total neutron-removal cross sections. This cross section exhibits a similar correlation with $L$ as the neutron-skin thickness [58].

\section{Conclusion}

Our understanding of the electric dipole response of exotic nuclei, in particular neutron-rich nuclei, has made substantial progress over the past about three decades since the first kinematically complete measurements of electromagnetic excitation at high beam energies have been performed. The second-generation experiments provided data with better statistics, in particular for halo nuclei. The characteristic low-energy response of one-neutron halo nuclei is meanwhile well understood and is used as a spectroscopic tool. The large cross section for Coulomb breakup and its extreme sensitivity to the halo effect provides an ideal tool also for exploratory studies of the most exotic systems produced only with very low beam intensities. Even the measurement of inclusive cross sections yields important information on the halo character and its structure, as recently demonstrated with the discovery of the heaviest halo nucleus ${ }^{31} \mathrm{Ne}$ in the region of the island of inversion. The first precise data for the dipole response of a two-neutron halo nucleus have been provided for ${ }^{11} \mathrm{Li}$ giving new insight into the response of barely bound threebody systems, and provide evidence for pronounced spatial neutron-neutron correlations in the ground-state of ${ }^{11} \mathrm{Li}$, as predicted by several three-body and other microscopic models. With the new next-generation experimental instruments like SAMURAI, already in operation at the RIBF at RIKEN, and $\mathrm{R}^{3} \mathrm{~B}$ starting operation at GSI, we can expect data with much improved precision, which will enable stringent tests of modern nuclear theories. 
Measurements of the electric dipole response of neutron-rich non-halo nuclei, and in particular of heavier nuclei, are still rather scarce. A general finding is a softening of the dipole response for neutron-rich nuclei. Resonance-like structures in the dipole response at around 9 to $10 \mathrm{MeV}$ have been observed for several nuclei, which are identified with the predicted low-energy pygmy resonance and understood as a dipole vibration of excess neutrons versus a core. But we are far away from a systematic investigation or understanding of the dipole response of neutron-rich nuclei. The next-generation experiments mentioned above have the potential for providing not only a systematic study but also much better precision of the extracted dipole-strength distributions. FAIR will extend the region of accessible nuclei substantially including heavy neutron-rich nuclei up to the lead region. Such measurements, covering the full energy range of the dipole response for heavier nuclei, will also extract the dipole polarizability of neutron-rich nuclei with good precision, which will provide important constraints on the equation of state of neutron-rich matter.

The author acknowledges support by the BMBF via Grant No. 05P15RDFN1, by GSI via the GSI-TU Darmstadt cooperation agreement, and support by the DFG via SFB1245.

Data Availability Statement This manuscript has no associated data or the data will not be deposited. [Author's comment: All data generated during this study are contained in this published article.]

Publisher's Note The EPJ Publishers remain neutral with regard to jurisdictional claims in published maps and institutional affiliations.

Open Access This is an open access article distributed under the terms of the Creative Commons Attribution License (http://creativecommons.org/licenses/by/4.0), which permits unrestricted use, distribution, and reproduction in any medium, provided the original work is properly cited.

\section{References}

1. P.F. Bortignon, A. Bracco, R.A. Broglia, Giant Resonances, Nuclear Structure at Finite Temperature (Harwood Acad., New York, 1998).

2. M.N. Harakeh, A. van der Woude, Giant Resonances: fundamental high-frequency modes of nuclear excitation (Clarendon Press, Oxford, 2001).

3. T. Aumann, P.F. Bortignon, H. Emling, Annu. Rev. Nucl. Part. Sci. 48, 351 (1998)

4. I. Tanihata, H. Hamagaki, O. Hashimoto, Y. Shida, N. Yoshikawa, K. Sugimoto, O. Yamakawa, T. Kobayashi, N. Takahashi, Phys. Rev. Lett. 55, 2676 (1985).

5. P.G. Hansen, B. Jonson, Europhys. Lett. 4, 409 (1987).

6. N. Paar, D. Vretenar, E. Khan, G. Colo, Rep. Prog. Phys. 70, 691 (2007).
7. S. Ebata, T. Nakatsukasa, T. Inakura, Phys. Rev. C 90, 024303 (2014).

8. D. Savran, T. Aumann, A. Zilges, Prog. Part. Nucl. Phys. 70, 210 (2013).

9. A. Bracco, E.G. Lanza, A. Tamii, Prog. Part. Nucl. Phys. 106, 360 (2019).

10. T. Aumann, T. Nakamura, Phys. Scr. T152, 014012 (2013).

11. P.-G. Reinhard, W. Nazarewicz, Phys. Rev. C 81, 051303 (2010).

12. X. Roca-Maza, N. Paar, Prog. Part. Nucl. Phys. 101, 96 (2018).

13. C.A. Bertulani, G. Baur, Phys. Rep. 163, 299 (1988).

14. T. Aumann, C.A. Bertulani, K. Sümmerer, Phys. Rev. C 51, 416 (1995).

15. E. Fermi, Z. Phys. 29, 315 (1924).

16. C.F. Weizsäcker, Z. Phys. 88, 612 (1934).

17. E.J. Williams, Phys. Rev. 45, 729 (1934).

18. A. Winther, K. Alder, Nucl. Phys. A 319, 518 (1979).

19. T. Aumann, Eur. Phys. J. A 26, 441 (2005).

20. D.M. Rossi, P. Adrich, F. Aksouh, H. Alvarez-Pol, T. Aumann, J. Benlliure, M. Böhmer, K. Boretzky, E. Casarejos, M. Chartier, A. Chatillon, D. Cortina-Gil, U. Datta Pramanik, H. Emling, O. Ershova, B. Fernandez-Dominguez, H. Geissel, M. Gorska, M. Heil, H. Johansson, A. Junghans, O. Kiselev, A. Klimkiewicz, J.V. Kratz, N. Kurz, M. Labiche, T. Le Bleis, R. Lemmon, Yu.A. Litvinov, K. Mahata, P. Maierbeck, A. Movsesyan, T. Nilsson, C. Nociforo, R. Palit, S. Paschalis, R. Plag, R. Reifarth, H. Simon, K. Sümmerer, A. Wagner, W. Walus, H. Weick, M. Winkler, Phys. Rev. Lett. 111, 242503 (2013).

21. H. Esbensen, B.A. Brown, H. Sagawa, Phys. Rev. C 51, 1274 (1995)

22. G. Gori, F. Barranco, E. Vigezzi, R.A. Broglia, Phys. Rev. C 69, 041302 (2004).

23. F. Barranco, G. Potel, R.A. Broglia, E. Vigezzi, Phys. Rev. Lett. 119, 082501 (2017).

24. T. Aumann, A. Navin, D.P. Balamuth, D. Bazin, B. Blank, B.A. Brown, J.E. Bush, J.A. Caggiano, B. Davids, T. Glasmacher, V. Guimarães, P.G. Hansen, R.W. Ibbotson, D. Karnes, J.J. Kolata, V. Maddalena, B. Pritychenko, H. Scheit, B.M. Sherrill, J.A. Tostevin, Phys. Rev. Lett. 84, 35 (2000).

25. R. Palit, P. Adrich, T. Aumann, K. Boretzky, B.V. Carlson, D. Cortina, Th.W. Elze, H. Emling, H. Geissel, M. Hellström, K.L. Jones, J.V. Kratz, R. Kulessa, Y. Leifels, A. Leistenschneider, G. Münzenberg, C. Nociforo, P. Reiter, H. Simon, K. Sümmerer, W. Walus, Phys. Rev. C 68, 034318 (2003).

26. T. Nakamura, S. Shimoura, T. Kobayashi, T. Teranishi, K. Abe, N. Aoi, Y. Doki, M. Fujimaki, N. Inabe, N. Iwasa, K. Katori, T. Kubo, H. Okuno, T. Suzuki, I. Tanihata, Y. Watanabe, A. Yoshida, M. Ishihara, Phys. Lett. B 331, 296 (1994).

27. N. Fukuda, T. Nakamura, N. Aoi, N. Imai, M. Ishihara, T. Kobayashi, H. Iwasaki, T. Kubo, A. Mengoni, M. Notani, H. Otsu, H. Sakurai, S. Shimoura, T. Teranishi, Y.X. Watanabe, K. Yoneda, Phys. Rev. C 70, 054606 (2004).

28. H.-W. Hammer, D.R. Phillips, Nucl. Phys. A 865, 17 (2011)

29. W. Nörtershäuser, D. Tiedemann, M. Zakova, Z. Andjelkovic, K. Blaum, M.L. Bissell, R. Cazan, G.W.F. Drake, Ch. Geppert, M. Kowalska, J. Krämer, A. Krieger, R. 
Neugart, R. Sanchez, F. Schmidt-Kaler, Z.-C. Yan, D.T. Yordanov, C. Zimmermann, Phys. Rev. Lett. 102, 062503 (2009)

30. S. Typel, G. Baur, Phys. Rev. Lett. 93, 142502 (2004).

31. D.J. Millener, J.W. Olness, E.K. Warburton, S.S. Hanna, Phys. Rev. C 28, 497 (1983).

32. P.G. Hansen, A.S. Jensen, B. Jonson, Annu. Rev. Nucl. Part. Sci. 45, 591 (1995).

33. T. Nakamura, T. Motobayashi, Y. Ando, A. Mengoni, T. Nishio, H. Sakurai, S. Shimoura, T. Teranishi, Y. Yanagisawa, M. Ishihara, Phys. Lett. B 394, 11 (1997).

34. T. Nakamura, N. Kobayashi, Y. Kondo, Y. Satou, N. Aoi, H. Baba, S. Deguchi, N. Fukuda, J. Gibelin, N. Inabe, M. Ishihara, D. Kameda, Y. Kawada, T. Kubo, K. Kusaka, A. Mengoni, T. Motobayashi, T. Ohnishi, M. Ohtake, N.A. Orr, H. Otsu, T. Otsuka, A. Saito, H. Sakurai, S. Shimoura, T. Sumikama, H. Takeda, E. Takeshita, M. Takechi, S. Takeuchi, K. Tanaka, K.N. Tanaka, N. Tanaka, Y. Togano, Y. Utsuno, K. Yoneda, A. Yoshida, K. Yoshida, Phys. Rev. Lett. 103, 262501 (2009).

35. T. Aumann, D. Aleksandrov, L. Axelsson, T. Baumann, M.J.G. Borge, L.V. Chulkov, J. Cub, W. Dostal, B. Eberlein, Th.W. Elze, H. Emling, H. Geissel, V.Z. Goldberg, M. Golovkov, A. Grünschloß, M. Hellström, K. Hencken, J. Holeczek, R. Holzmann, B. Jonson, A.A. Korshenninikov, J.V. Kratz, G. Kraus, R. Kulessa, Y. Leifels, A. Leistenschneider, T. Leth, I. Mukha, G. Münzenberg, F. Nickel, T. Nilsson, G. Nyman, B. Petersen, M. Pfützner, A. Richter, K. Riisager, C. Scheidenberger, G. Schrieder, W. Schwab, H. Simon, M.H. Smedberg, M. Steiner, J. Stroth, A. Surowiec, T. Suzuki, O. Tengblad M.V. Zhukov, Phys. Rev. C 59, 1252 (1999).

36. T. Nakamura, A.M. Vinodkumar, T. Sugimoto, N. Aoi, H. Baba, D. Bazin, N. Fukuda, T. Gomi, H. Hasegawa, N. Imai, M. Ishihara, T. Kobayashi, Y. Kondo, T. Kubo, M. Miura, T. Motobayashi, H. Otsu, A. Saito, H. Sakurai, S. Shimoura, K. Watanabe, Y.X. Watanabe, T. Yakushiji, Y. Yanagisawa, K. Yoneda, Phys. Rev. Lett. 96, 252502 (2006).

37. F. Barranco, P.F. Bortignon, R.A. Broglia, G. Colo, E. Vigezzi, Eur. Phys. J. A 11, 85 (2001).

38. H. Simon, D. Aleksandrov, T. Aumann, L. Axelsson, T. Baumann, M.J.G. Borge, L.V. Chulkov, R. Collatz, J. Cub, W. Dostal, B. Eberlein, Th.W. Elze, H. Emling, H. Geissel, A. Grünschloß, M. Hellström, J. Holeczek, R. Holzmann, B. Jonson, J.V. Kratz, G. Kraus, R. Kulessa, Y. Leifels, A. Leistenschneider, T. Leth, I. Mukha, G. Münzenberg, F. Nickel, T. Nilsson, G. Nyman, B. Petersen, M. Pfützner, A. Richter, K. Riisager, C. Scheidenberger, G. Schrieder, W. Schwab, M.H. Smedberg, J. Stroth, A. Surowiec, O Tengblad, M.V. Zhukov, Phys. Rev. Lett. 83, 496 (1999).

39. J. Tanaka, R. Kanungo, M. Alcorta, N. Aoi, H. Bidaman, C. Burbadge, G. Christian, S. Cruz, B. Davids, A. Diaz Varela, J. Even, G. Hackman, M.N. Harakeh, J. Henderson, S. Ishimoto, S. Kaur, M. Keefe, R. Krücken, K.G. Leach, J. Lighthall, E. Padilla Rodal, J.S. Randhawa, P. Ruotsalainen, A. Sanetullaev, J.K. Smith, O. Workman, I. Tanihata, Phys. Lett. B 774, 268 (2017).

40. H. Esbensen, K. Hagino, P. Mueller, H. Sagawa, Phys. Rev. C 76, 024302 (2007).

41. S. Funada, H. Kameyama, Y. Sakuragi, Nucl. Phys. A 575 93 (1994).
42. L.-B. Wang, P. Mueller, K. Bailey, G.W.F. Drake, J.P. Greene, D. Henderson, R.J. Holt, R.V.F. Janssens, C.L. Jiang, Z.-T. Lu, T.P.O. Connor, R.C. Pardo, K.E. Rehm, J.P. Schiffer, X.D. Tang, Phys. Rev. Lett. 93, 142501 (2004).

43. G. Papadimitriou, A.T. Kruppa, N. Michel, W. Nazarewicz, M. Poszajczak, J. Rotureau, Phys. Rev. C 84, 051304 (2011).

44. T. Aumann, T. Nakamura, Dipole response of the dripline nuclei ${ }^{6,8} \mathrm{He},{ }^{24} \mathrm{O}$, and ${ }^{29} \mathrm{~F}$ : Search for the genuine soft dipole mode, proposal for an experiment at RI Beam Factory (2015).

45. J. Gibelin, D. Beaumel, T. Motobayashi, Y. Blumenfeld, N. Aoi, H. Baba, Z. Elekes, S. Fortier, N. Frascaria, N. Fukuda, T. Gomi, K. Ishikawa, Y. Kondo, T. Kubo, V. Lima, T. Nakamura, A. Saito, Y. Satou, J.-A. Scarpaci, E. Takeshita, S. Takeuchi, T. Teranishi, Y. Togano, A.M. Vinodkumar, Y. Yanagisawa, K. Yoshida, Phys. Rev. Lett. 101, 212503 (2008).

46. A. Leistenschneider., T. Aumann, K. Boretzky, D. Cortina, J. Cub, U. Datta Pramanik, W. Dostal, Th.W. Elze, H. Emling, H. Geissel, A. Grünschloß, M. Hellström, R. Holzmann, S. Ilievski, N. Iwasa, M. Kaspar, A. Kleinböhl, J.V. Kratz, R. Kulessa, Y. Leifels, E. Lubkiewicz, G. Münzenberg, P. Reiter, M. Rejmund, C. Scheidenberger, Ch. Schlegel, H. Simon, J. Stroth, K. Sümmerer, E. Wajda, W. Walus, S. Wan, Phys. Rev. Lett. 86, 5442 (2001).

47. P. Adrich, A. Klimkiewicz, M. Fallot, K. Boretzky, T. Aumann, D. Cortina-Gil, U. Datta Pramanik, Th.W. Elze, H. Emling, H. Geissel, M. Hellström, K.L. Jones, J.V. Kratz, R. Kulessa, Y. Leifels, C. Nociforo, R. Palit, H. Simon, G. Surówka, K. Sümmerer, W. Walus, Phys. Rev. Lett. 95, 132501 (2005).

48. LAND Collaboration (A. Klimkiewicz, N. Paar, P. Adrich, M. Fallot, K. Boretzky, T. Aumann, D. Cortina-Gil, U. Datta Pramanik, Th.W. Elze, H. Emling, H. Geissel, M. Hellström, K.L. Jones, J.V. Kratz, R. Kulessa, C. Nociforo, R. Palit, H. Simon, G. Surowka, K. Sümmerer, D. Vretenar, W. Walus), Phys. Rev. C 76, 051603 (2007).

49. S. Bacca, N. Barnea, G. Hagen, M. Miorelli, G. Orlandini, T. Papenbrock, Phys. Rev. C 90, 064619 (2014).

50. O. Wieland, A. Bracco, F. Camera, G. Benzoni, N. Blasi, S. Brambilla, F.C.L. Crespi, S. Leoni, B. Million, R. Nicolini, A. Maj, P. Bednarczyk, J. Grebosz, M. Kmiecik, W. Meczynski, J. Styczen, T. Aumann, A. Banu, T. Beck, F. Becker, L. Caceres, P. Doornenbal, H. Emling, J. Gerl, H. Geissel, M. Gorska, O. Kavatsyuk, M. Kavatsyuk, I. Kojouharov, N. Kurz, R. Lozeva, N. Saito, T. Saito, H. Schaffner, H.J. Wollersheim, J. Jolie, P. Reiter, N. Warr, G. deAngelis, A. Gadea, D. Napoli, S. Lenzi, S. Lunardi, D. Balabanski, G. LoBianco, C. Petrache, A. Saltarelli, M. Castoldi, A. Zucchiatti, J. Walker, A. Bürger, Phys. Rev. Lett. 102, 092502 (2009).

51. O. Wieland, A. Bracco, F. Camera, R. Avigo, H. Baba, N. Nakatsuka, T. Aumann, S.R. Banerjee, G. Benzoni, K. Boretzky, C. Caesar, S. Ceruti, S. Chen, F.C.L. Crespi, V. Derya, P. Doornenbal, N. Fukuda, A. Giaz, K. Ieki, N. Kobayashi, Y. Kondo, S. Koyama, T. Kubo, M. Matsushita, B. Million, T. Motobayashi, T. Nakamura, M. Nishimura, H. Otsu, T. Ozaki, A.T. Saito, H. Sakurai, H. Scheit, F. Schindler, P. Schrock, Y. Shiga, M. Shikata, S. Shimoura, D. Steppenbeck, T. Sumikama, S. Takeuchi, R. Taniuchi, Y. Togano, J. Tscheuschner, J. Tsubota, H. 
Wang, K. Wimmer, K. Yoneda, Phys. Rev. C 98, 064313 (2018).

52. X. Roca-Maza, M. Brenna, G. Colò, M. Centelles, X. Vinas, B. K. Agrawal, N. Paar, D. Vretenar, J. Piekarewicz, Phys. Rev. C 88, 024316 (2013).

53. J. Piekarewicz, Phys. Rev. C 73, 044325 (2006).

54. X. Roca-Maza, X. Vinas, M. Centelles, B.K. Agrawal, G. Colò, N. Paar, J. Piekarewicz, D. Vretenar, Phys. Rev. C 92, 064304 (2015).

55. A. Tamii, I. Poltoratska, P. vonNeumann-Cosel, Y. Fujita, T. Adachi, C.A. Bertulani, J. Carter, M. Dozono, H. Fujita, K. Fujita, K. Hatanaka, D. Ishikawa, M. Itoh, T. Kawabata, Y. Kalmykov, A.M. Krumbholz, E. Litvinova, H. Matsubara, K. Nakanishi, R. Neveling, H. Okamura, H.J. Ong, B. Ozel-Tashenov, V.Y. Ponomarev, A. Richter, B. Rubio, H. Sakaguchi, Y. Sakemi, Y. Sasamoto, Y. Shimbara, Y. Shimizu, F.D. Smit, T. Suzuki, Y. Tameshige, J. Wambach, R. Yamada, M. Yosoi, J. Zenihiro, Phys. Rev. Lett. 107, 062502 (2011).
56. T. Hashimoto, A.M. Krumbholz, P.-G. Reinhard, A. Tamii, P. von Neumann-Cosel, T. Adachi, N. Aoi, C.A. Bertulani, H. Fujita, Y. Fujita, E. Ganioglu, K. Hatanaka, E. Ideguchi, C. Iwamoto, T. Kawabata, N.T. Khai, A. Krugmann, D. Martin, H. Matsubara, K. Miki, R. Neveling, H. Okamura, H.J. Ong, I. Poltoratska, V.Yu. Ponomarev, A. Richter, H. Sakaguchi, Y. Shimbara, Y. Shimizu, J. Simonis, F.D. Smit, G. Süsoy, T. Suzuki, J.H. Thies, M. Yosoi, J. Zenihiro, Phys. Rev. C 92, 031305 (2015).

57. A. Carbone, G. Colò, A. Bracco, L.-G. Cao, P.F. Bortignon, F. Camera, O. Wieland, Phys. Rev. C 81, 041301 (2010).

58. T. Aumann, C.A. Bertulani, F. Schindler, S. Typel, Phys. Rev. Lett. 119, 262501 (2017). 